\title{
Research on the English Translation of Luzhou Intangible Cultural Heritage Names in the Memetics Perspective
}

\author{
Li Xiao ${ }^{1, a}$ \\ School of Foreign Languages, Southwest Medical University, Lu Zhou Si Chuan, China, (E-mail: \\ xiaoli60711@163.com)
}

\begin{abstract}
Keywords: English Translation, Luzhou Intangible Cultural Heritage Names, Memetics Perspective Project: 1. Research on the English Translation of Luzhou Intangible Cultural Heritage Names in the Memetics Perspective (NO: LZ16A95)

2. Research on the Foreign Propaganda Text's English Translation of Si Chuan Intangible Cultural Heritage in the Memetics Perspective (NO: LYC16-37 )
\end{abstract}

\begin{abstract}
Luzhou has a large number of non-material cultural heritage, reflecting the ancient history and culture of the essence, unique heritage and charming, to bring to the world, the name of the English translation of the intangible cultural heritage is appropriate or not of critical importance. Translator translation process involves decoding the source language memes and memes with goals two re-encoding process. In the source language and the target language meme to build a translation of the library has a variety of memes relationship and they interact to promote the spread and development of cultural memes. According to this classification will be classified and analyzed Luzhou name, help better tap the name of the attraction behind profound cultural connotations, to promote cross-cultural development.
\end{abstract}

\section{Introduction}

"Intangible cultural heritage" means "being of communities, groups and, sometimes, individuals recognize as various social part of their cultural heritage, the concept of representation , expressions, knowledge, skills and related instruments, objects, artefacts and cultural spaces. "' non-left "known reputation" national culture root ", it embodies a kind of" local knowledge "of cultural values and historical heritage, which is an important carrier of human wisdom and civilization, its significance mainly for empirical tradition, concentrated and highly personalized nationality.

Memetics is based on the Darwinian point of view, a new theory attempts to reveal the evolution of human culture, heritage laws. Center is the term meme theory. In 1976, the famous Oxford University zoologist Richard Dawkins wrote, "The Selfish Gene", exploring gene replicate itself and competes with each other on the promotion of basic biological evolution, first proposed memes (meme). Meme is called "unit or units of cultural transmission imitation" meme is through imitation and replication between the human brain and spread the infection to each other. Later, in the masterpiece "meme machine" in, Susan Blackmore memes proposed mechanism of operation is similar to running genes. He insisted that: between memes are competing, selfish, desperate to enter into another person's brain, another book, among other objects, and the final decision of our cultural and psychological structure. From this process, memes replicate the results are not necessarily consistent, equivalent to more non-transplant sense, the process of transmission of meme is a kind of "Unequal" process. It is this meme role in the formation of the local culture, but also to promote communication and exchange between cultures, the formation of cross-cultural. In fact, the translation process is essentially a foreign language through cultural memes to their propagation process. Therefore, we proceed from the memetics perspective to explain translation can better grasp the essence of cross-cultural translation to some extent.

\section{Meme and Translation}

In the historical development of meme, the meme system proposed relationship between translation theories is the Finnish scholar Chesterman. He put in the translation process of the formation of 
concepts or ideas on translation and translation theory referred to as translation memes, as some translate theoretical concepts, norms, policies and values. There are five super meme: source language - target language memes peer meme, meme untranslatable, free translation - literally memes, memes writing that is translated.

If a culture unit has been spread, it became meme. Similarly, when a language unit obtained in different locales spread, it became the translation of memes that get cultural memes spread through an interpreter. Thus, the meme is the basic unit of cultural translation and the internal operating mechanism memes determine the specific operation of translation. How natural positive thought "meme theory is to explain the new theory of cultural evolution law", the translation of the meme can be used to explain and promote the mutual exchange between the cultures of each other law.

\section{Translation Process of the Propagation Memes}

According FrancisHeylighen theory, memes propagate need to get through four stages: assimilation, memory, expression and communication. Assimilation refers to the so-called memes are presented receptor attention, understanding and acceptance. When a meme receptor caused concern, and has been accepted on the basis of understanding in order to be included in their cognitive system and then get stored in the memory. Saved memes must be translated by a memory mode for the audience to be able to perceive the corporeal, that is, through sound, images or language "expression" to pass to another receptor, resulting in the spread. Thus, cultural memes really established.

And due to the construction of cultural molding process is similar to the translation of a meme is the assimilation, memory, expression and ultimately intercultural communication process. For the translator, a good source is first author coded memes, then the translator as a receptor according to their existing cognitive system decoding the source language author of memes, and assimilated into their cognitive system. Then, the translator and assume the role of the body to assimilate the target of memes with memes recode express and disseminate to the reader, the reader is the translator of recoding memes according to their cognitive system to decode, then further spread to other people. This process involves two codes, decoded twice. The translation of meme propagation are mainly concentrated in the translator and the translator 1 decoding encoding step 2. If the translator does not know the author of the source language and memes meaning, you cannot correctly decode the same time, if the translator is not proficient in the target language readers' memes, could not be better coding and thus serve as an effective spread. Therefore, the translation of the meme spread mainly due to the translator for source and target languages and cultural understanding and application. Translator memes both exotic and decoding of those infected, but also an important disseminator of exotic memes.

Translation of memes propagate through the final forming, in fact, is a sense in terms of memes, embodied as the source language and the target language meme two forms. If a source memes successful transition into the target language memes, the translation of memes are successful, meaning they process through the dissemination of culture in the two fields of communication and implementation.

How to ensure the translation of meme propagation of memes semantic translation in the source language and the target under two forms memes consistency, which is to ensure the source language and the target language memes in semantic reciprocity is the real problem to be solved.

\section{Luzhou Intangible Cultural Heritage}

Luding County is in southern Sichuan and it has a long history and profound culture, it is the one of one hundred thousand ancient counties, the hometown of Chinese dragon culture, rich folk culture resources. Beach has a mysterious lion, month mother chicken soup, dragon altar rain and other non-heritage items.

Fantasy Luzhou pool wine traditional brewing process contains a wealth of historical, scientific, cultural and economic value and it is a Chinese folk tradition of craftsmanship of many highlights. It is not only the rich heritage context of Luzhou wine, its art creation, development and growth 
history is a microcosm of the history of civil Luzhou liquor and local economic and social development. Even in science developed today, it is difficult to give instead of using scientific means. It is a treasure trove of traditional folk art of brewing in a wonderful work and the ancestors left us a precious heritage.

Luzhou paper umbrella is one of Han Chinese folk crafts. Luzhou City, Sichuan Basin is located in the Yunnan-Guizhou Plateau over with adequate rainfall, abundant bamboo resources, with 400 years of history paper umbrella manufacturing production in 2008 Jiangyang government "Luzhou paper umbrella production process," declared as "national intangible of cultural heritage ", by experts as" Chinese folk arts umbrella living fossil. " A paper umbrella repeated 3,000 times without damaging the income support, soaked in water for 24 hours off the bone, the top five wind umbrella walking without deformation. As currently the only one fully retains all handmade of intangible cultural heritage, Luzhou paper umbrella is almost an exhaustive inherited the traditional Chinese umbrella skills. According to the production process in the watershed paper umbrella at the junction of Sichuan, Guizhou and the sixth generation of successors completed the six-rich presentation:. "End production of an umbrella, even the experienced master craftsmen also need two weeks" is expected to open to play from production completed an umbrella to go through 90 procedures, use hundreds of tools. "The key is to have to see the weather, the sun not big, nor rainy." Climate primarily affects rib forming and paper flat, curved or resulting from the shell. Since successfully declared a national non-heritage projects, watershed paper umbrella plant Umbrella tall century-old house yard, full of bamboo, colored lines and semi-finished products umbrella, thick tung oil smell in the air.

\section{Categories of Memes Relations}

The most difficult in translation is the noun phrase. Attractions Name tourists contact first scenery, the landscape itself is their first impression, tourist attractions is the crystallization of national culture, foreign friends understand China's splendid culture "bridge." Attractions witness the history of precipitation, the years and the touchstone of national development. Attractions name translation to fully reflect its short and pithy, easy to remember characteristics.

Anthropomorphic Meaning Relations. The so-called anthropomorphic meaning relationship is the source language and the target language memes in form and semantics are basically the same. Form here is the syntactic form or voice form. Mold such relationships because of their semantic equivalence highest, and therefore most likely to be spread, most likely to form the translation of the meme. This translation of the meme phenomenon generally occurs in the codification bilingual dictionary or a translation of some terms. Although it is a simple way to spread memes, memes propagate culture is the most basic and most common way. Therefore, this translation of memes propagate is easier, but also to build a foundation of human cultural transmission.

Alien Synonymous Relationship. Due to this type of relationship in the translation process is the most common. Because, people's living environment is almost always those people and those things, but the language once formed it will have its own operating mechanism, which makes synonymous but alien memes most common. Therefore, when the translation of the spread of memes, need to use different forms to express the same semantics.

English is the "Forms" language, structured syntax, grammar role interlocking, logical, and Chinese is "Ideas", shape evacuated grammatical role and great flexibility. In this cultural context, Westerners heavy rationality, logic, evidence, but Asians but heavy savvy, intuition, imagery. Although formally belong profiled synonymous, but still be able to easily communicate with each other in understanding, to understand the environment of mutual bright, easy to arouse cultural induction, enabling the translation of memes spread.

Meaning Nearly Alien Relationship. In the translation process, some source memes can happen because of them find a similar expression in the target language mode, but not in the full sense of semantics, etc., but the extended meaning and so on, that is, Although it expressed by different things, but the idea is basically the same meaning. We call this relationship between memes called profiled nearly meaning, common in the idiom or idiomatic expression. 
To ensure that the target language readers understand the basis, we can use translation method of alienation. As a result, alienation translation can enrich the target language expressions, the source language memes promote exchanges and closer to the target language and memes, memes enhanced vitality. The translation of memes, lifelike images, haunting, to control people's things underworld and ability have been very vivid. This will not only convey the intent of the original message, but also the flexibility to reproduce the author's communicative intent; not only fascinating and Western tourists "face" communication, but also to meet their psychological self-esteem, evoke positive psychological sense, the attraction to perfection manifested reads very fluent, in order to better achieve the translation of memes propagate, thus achieving the intended purpose of tourism translation.

No Justice Relationship. Sometimes the source language in the target language memes meme library which can not find peer semantic memes, we will call no Memes such as justice relations. This non-relationship, such as justice is actually the opposite. As long as we are able to target memes library renewable create a meme to the source language memes such as justice, and gradually get the public to accept this, such as justice and human relations become. The successful creation of the target language memes, such as taking into account sound, form, meaning, it can greatly facilitate this translation of intercultural communication memes.

Above four source and target languages in relations memes actual translation process are synonymous with the pursuit of the goal. Anthropomorphic meaning relationships are so; profiled synonyms semantic relations required to maintain consistency in the case of a formal accord; profiled close relationship also requires justice as long as the recipient is decoded, it is possible by means of alienation in pursuit of source and target synonymous as the goal; no relationship, such as justice, after the establishment of the target language, synonymous relations among people gradually formed in the cognitive system. Cultural Loss refers to a national language unique cultural connotation failed to express or not fully expressed.

\section{Conclusion}

If you get any of the cultural institutions of intercultural communication through an interpreter, it would be translated of memes. Translation of the meme source language and the target language memes two forms in the sense of unity together. The source language and the target language meme together constitute the translation of the library, which is the principle of construction of semantic linking. In this meme library, a relationship between the various memes, synonymous with the pursuit, including translation and cultural Meaning of the goal, and also avoid the phenomenon of loss of cultural transmission. Luzhou attractions will be analyzed according to the name of this classification method, obtain a new version of the translation, the ultimate goal is the interpretation of the name behind the deep-seated culture and heritage, the promotion of relations between the various memes interaction and development, promotion of culture dissemination and exchange.

\section{References}

[1] Huifang Zhou: Linguistic Sciences, Vol. 6 (2014) No 53, p.25-26

[2] Hongli Zhang: Central South University, Vol. 12 (2015) No 27, p.74-76

[3] Qin Guo: Journal of Personality and Social Psychology, Vol. 1 (2011) No 33, p.11-14

[4] Jieming Liu: Guangxi Normal University, Vol. 3 (2012) No33, p.121-124

[4] Wang Jun: The Translator, Vol. 3 (2009) No47, p.58-60 